\title{
Reply to "Comment on 'Photoionization of helium atoms irradiated with intense vacuum ultraviolet free-electron laser light. Part II. Theoretical modeling of multiphoton and single-photon processes" "
}

\author{
A. R. B. de Castro, ${ }^{1,2}$ T. Laarmann, ${ }^{2}$ J. Schulz, ${ }^{2}$ H. Wabnitz, ${ }^{2}$ and T. Möller ${ }^{2}$ \\ ${ }^{1}$ Laboratorio Nacional de Luz Sincrotron LNLS, 13084-971 Campinas SP, Brazil and Instituto de Fisica Gleb Wataghin, \\ Universidade Estadual de Campinas IFGW-UNICAMP, 13083-970 Campinas SP, Brazil \\ ${ }^{2}$ Hamburger Synchrotronstrahlungslabor HASYLAB at Deutsches Elektronen Synchrotron DESY, Notkestr. 85, 22607 Hamburg, Germany
}

(Received 26 May 2006; published 1 August 2006)

\begin{abstract}
We cannot agree with the Comment by Maquet et al. [Phys. Rev. A. 74, 027401 (2006)]. It seems that Maquet et al. are thinking in terms of classical fields. In our recent paper we have presented a study of the two-photon rate of He making use of quantized fields. This approach becomes a natural choice if we have in mind processes at high photon energy. In this description of the problem the squared vector potential $\mathbf{A} \cdot \mathbf{A}$ term is essential.
\end{abstract}

DOI: 10.1103/PhysRevA.74.027402

PACS number(s): 42.50.Hz, 33.60.Cv, 32.80.Rm

First of all, we would like to make a comment on the validity of perturbation treatments. Let $\gamma$ be the Keldysh parameter and $F$ the field strength. We all agree that, for fixed ionization energy $I_{\mathrm{P}}$ and field frequency $\omega$, a perturbative regime will be reached in the limit $\gamma \rightarrow \infty(F \rightarrow 0)$, while the perturbation theory will break down in the opposite limit $\gamma$ $\rightarrow 0(F \rightarrow \infty)$. Yet, the value of $\gamma$ cannot be the only guideline as to the applicability of the perturbation theory. The Keldysh paper [1] had in mind ir-visible lasers, considered uniform electric fields only, discarded the $\mathbf{A} \cdot \mathbf{A}$ term, was based on free-electron wave functions in the presence of uniform fields, and was basically a perturbation calculation itself. As a result, at short wavelength, especially if we think about $\mathrm{x}$ rays, the concept of the Keldysh parameter gives scanty insight on whether the perturbation theory can be applied or not. Finally, calculations using the Fermi golden rule do not preclude a more detailed analysis, such as the one we believe to have presented.

On very general grounds we can state that the use of a complete nonorthogonal set of quantum basis states does not pose any fundamental restriction on the solution of a quantum mechanical problem. However, the relative importance of contributions from various terms in the Hamiltonian depends on the basis. For our basis set, only $\mathbf{A} \cdot \mathbf{A}$ connects the initial $|1 s 1 s\rangle|N\rangle$ and final $\left|1 s(Z=2) \mathbf{k}_{\text {electron }}\right\rangle|N-2\rangle$ states involved in the two-photon transition, because our photon states are exact energy eigenstates of the radiation field. To say it more clearly, if the $\mathbf{A} \cdot \mathbf{A}$ term is omitted, there is no two-photon transition at all.

The counter argument given by Maquet et al. ("any solution $\Psi(t)$ of the TDSE with the $A^{2}(t)$ term is related to the solution $\Phi(t)$, without $A^{2}(t)$ through $\left.\Psi(t)=\exp \left[-i \int_{0}^{t} A^{2}(u) d u\right] \Phi(t), \ldots,\right) \quad$ is quite fallacious. As a matter of fact, if $i \hbar \partial \Psi / \partial t=\{H+f(t)\} \Psi$, if we define $U=\exp \left[-i \int_{0}{ }^{\mathrm{t}} f(u) d u / \hbar\right]$ and $\Psi=U \Phi$, then, quite generally, $i \hbar \partial \Phi / \partial t=\left\{U^{-1} H U\right\} \Phi$. It is only when $H$ and $U$ commute that $U^{-1} H U=H$ and the result quoted by Maquet et al. is valid. In our case, since we adopt a quantum description of all fields, both $H$ and $U$ depend on the photon creation and annihilation operators and do not commute at all. We have, of course, checked that the overlap matrix is correctly taken into ac- count and that, in the absence of external laser field, the system remains in the initial state, to a precision of 1 part in $10^{7}$ in the occupation probabilities.

Let us also state that the electric dipole approximation is in no way an essential feature of our calculation. As a matter of fact, it is expected to be invalid for experiments with the hard x-ray lasers presently under development. However, it was convenient for two reasons. First, $k_{\mathrm{rad}} R$ ( $R=$ some effective size of the He atom) was not large in our experiments; second, we estimate that errors incurred in the truncation of the basis set and in the approximate nature of the He eigenstates are the limiting factors in the quality of our calculation. Should exact energy eigenstates $\left|\Psi_{\mathrm{j}}\right\rangle$ for He become available, they would automatically satisfy $\left\langle\Psi_{\mathrm{i}} \mid \Psi_{\mathrm{j}}\right\rangle=0$ if $E_{\mathrm{i}}$ $\neq E_{\mathrm{j}}$. It is obvious that then, in the electric dipole moment approximation (spatially uniform $\mathbf{A}$ ), $\left\langle\Psi_{\mathrm{i}}|\mathbf{A} \cdot \mathbf{A}| \Psi_{\mathrm{j}}\right\rangle=0$ if $\left\langle\Psi_{\mathrm{i}} \mid \Psi_{\mathrm{j}}\right\rangle=0$, as observed by Maquet et al. The exact $\mathbf{A} \cdot \mathbf{A}$ interaction, however, which should be dominant at shorter wavelengths and larger field intensities, has a spacedependent factor $\exp \left( \pm 2 i \mathbf{k}_{\mathrm{rad}} \cdot \mathbf{r}\right)$ and now the Cohan Haneka theorem [2] can no longer be invoked to guarantee that all off-diagonal elements vanish.

Regarding the Schmidt orthogonalization of the approximate He eigenstates available in the literature (and used in our calculation), let us observe that this procedure mixes in states of different energy. Were we to orthogonalize the $\mathrm{He}$ ground state $|1 s 1 s\rangle$ and the state $\left|1 s(Z=2) \mathbf{k}_{\text {eletr }}\right\rangle$ related to the two-photon transitions under discussion, we should for consistency also orthogonalize the state $|1 s 2 s\rangle$ with regard to $|1 s 1 s\rangle$. This would result in a shift of the expected value $\langle 1 s 2 s|H| 1 s 2 s\rangle$. Then it becomes legitimate to ask whether the orthonormalized but spectrally shifted states are a better approximation of the He exact eigenstates at all, given the exquisite agreement of the energies derived from the He states used in our calculation and both the "reference" calculation [3] and experimentally measured spectra [4].

Still in this connection (too few functions in the helium Hilbert space) we should like to thank Maquet et al. for calling our attention to such treacherous pitfalls. However, consider: we approximated each $\mathrm{He}$ eigenstate with some linear combination of about 60 
functions $\chi\left(\mathbf{r}_{1}, \mathbf{r}_{2}\right)=r_{1}{ }^{\mathrm{m}} r_{2}{ }^{\mathrm{n}} r_{12}{ }^{\mathrm{q}} \exp \left(-a r_{1}-b r_{2}-c r_{12}\right)$, where $r_{12}=\left|\mathbf{r}_{1}-\mathbf{r}_{2}\right|$ and $n, m, q=0,1,2, \ldots$. The factor $\exp \left(-c r_{12}\right)$ is equivalent to an infinite sum involving products of the spherical harmonic functions $Y_{\lambda \mu}{ }^{*}\left(\theta_{1} \phi_{1}\right) Y_{\lambda \mu}\left(\theta_{2} \phi_{2}\right)$. Our orbital basis is therefore seen to include the same one-particle states and the same correlated factors $r_{12}$ as in Ref. [5], except insofar as Scrinzi and Piraux used only a finite set of such one-particle states.

The $\mathbf{E} \cdot \mathbf{r}$ interaction is equivalent to $\mathbf{p} \cdot \mathbf{A}$ only for uniform electric fields. Although we saw no cogent reason to invoke $\mathbf{E} \cdot \mathbf{r}$ in our calculation, we did estimate the accuracy of our relevant matrix elements comparing the numerical values of $\left\langle\right.$ final $\left|p_{z}\right|$ initial $\rangle$ and $m\left(E_{\text {final }}-E_{\text {initial }}\right)\langle$ final $|z|$ initial $\rangle$. Quite independently of electromagnetic gauge transformations, these two quantities should be equal for exact eigenstates of our He Hamiltonian. We found a discrepancy of about $40 \%$, which we take as an indication on the accuracy of the absolute values of the transition probability calculated in the pa- per. However, since this is a systematic error related to the He wave functions, we believe that the dependence of the transition probabilities on radiant field intensity $I$ is affected to a much lesser degree, roughly speaking, by an overall factor slowly dependent on I. By the way, no error estimate is given in the calculation of Ref. [5]. It has not yet been possible to measure absolute transition probabilities in the experiments reported in our Physical Review A papers, but we do believe that final validation of any proposed theoretical model rests on a detailed comparison with experiment.

In conclusion, there is no doubt that the quantized field approach used in our paper, where the $\mathbf{A} \cdot \mathbf{A}$ term is responsible for two-photon transitions, is legitimate. On the other hand we can agree that the $\mathbf{A} \cdot \mathbf{A}$ term might play only a minor role on theoretical work based on classical fields, such as Ref. [5]. Further theoretical work and comparison with experiments can help to clarify this point.
[1] L. V. Keldysh, Sov. Phys. JETP 20, 1307 (1965).

[2] N. V. Cohan and H. Hameka, Phys. Rev. 151, 1076 (1966).

[3] A. J. Thakkar and V. H. Smith, Phys. Rev. A 15, 1 (1977); Phys. Rev. A 15, 16 (1977), and references herein.
[4] Visit physics.nist.gov/PhysRefData/, Atomic Spectroscopy Data, Atomic Spectra Database v3.0, He I. A table of energy levels for He I and a list with more than 40 supporting references is given there.

[5] A. Scrinzi and B. Piraux, Phys. Rev. A 58, 1310 (1998). 